\title{
PARTICIPATION OF MEMBRANE NANOTUBES IN INTERCELLULAR COMMUNICATION
}

\author{
Agnieszka KNOPIK-SKROCKA, Agata ŚNIEGOWSKA
}

Department of Cell Biology, Adam Mickiewicz University of Poznań

\author{
DOI: $10.2478 / \mathrm{acb}-2014-0004$
}

\begin{abstract}
Summary: A few years ago, a so far unknown type of intercellular connections, involved in communication was discovered. Due to their specific nano-architecture, these connections were named membrane nanotubes or tunneling nanotubes. Nanotubes ensure the transfer of both membrane and cytosolic cellular components, including organelles. Nanotubes also participate in calcium signal transduction and apoptosis signal. The length of the distance at which cells contact via nanotubes reaches several hundred micrometers. The published data suggest that nanotubes have heterogeneous structure. Among them, there are nanotubes which provide direct contact of the cytoplasm in connected cells (open-ended structure), and those, in which the transport requires overcoming a barrier, which is the cell membrane (close-ended structure). An important finding in the study of membrane nanotubes was demonstrating the ability of these connections of the intercellular transfer of pathogens, such as HIV, or abnormal form of PrP prion protein. In addition, nanotubes mediate a transport of MDR protein, involved in resistance of cancer cells to chemotherapy. It means that this type of cell connection may play an important role in the pathomechanism of AIDS, prion as well as cancer diseases.
\end{abstract}

Key words: membrane nanotubes, intercellular communication, organelle transport, signal transduction, transmission of pathogens

Abbreviations: ABC ATP-binding cassette transporters; Akt3 RAC-gamma serine/threonine-protein kinase; ALPS autoimmune lymphoproliferative syndrome; anti-EEA1 early endosome autoantigen 1 antibody; CD4 cluster of differentiation 4; CD59 cluster of differentiation 59; CDC42 cell division control protein 42; DC-STAMP dendritic cell-specific transmembrane protein; DiD 11 'dioctadecyl-3, 3, 3`3'-tetramethylindodicarbocyanine perchlorate; DiO dioctadecyloxacarbocyanine perchlorate ; EPC endothelial progenitor cells; GFP green fluorescent protein; GM1 monosialotetrahexosylganglioside; GPCR G protein coupling receptors; GPI glycosyl-phosphatidylinositol; HEK293T human embryonic kidney cells; HIV human immunodeficiency virus; HUVEC human umbilical vein endothelial cells; I-BAR Bin-amphiphysin-Rvs superfamily proteins; m- $\boldsymbol{\beta} \mathbf{C D}$ methyl- $\beta$-cyclodextrin; MDR multidrug resistance protein; MHC major histocompatibility complex; MLV murine leukemia virus; MMSC multipotent stromal cells; MSCs mesenchymal stem cells; M-Sec tumor necrosis factor- $\alpha$ induced protein 2 (BP94); MSTO-211H human malignant pleural mesothelioma; mTOR mam- 
malian target of rapamycin; NK natural killers; PC12 rat pheochromocytoma cells; PI3K phosphoinositide 3-kinase; PrP prion protein; PrPsc scrapie prion protein; QGY-7703 human hepatocellular carcinoma cells; RaIA Ras-related small GTPase; Rho Ras homologous protein family; RTC renal tubular cells; RT4 cell line derived from a transitional epithelium of urinary bladder; SH-SY5Y neuroblastoma; T24 transitional cell carcinoma of urinary bladder; TNT tunneling nanotubes.

\section{INTRODUCTION}

Intercellular communication is a fundamental phenomenon that provides control and integration of physiological processes in living organism [46]. Plasmodesmata, discovered over 100 years ago, are involved in information exchange and cytoplasm connection between plant cells $[5,17,58]$. These channels include actin filaments and so-called desmotubule, which passes through the center of plasmodesmata. Desmotubule is a part of the modified endoplasmic reticulum ER.

Secretion of signal molecules outside the cell and their transmission to the target cells (mainly by paracrine and hemocrine) is a classic mechanism of intercellular communication in animals [35]. Microvesicles, exosomes as well as ectosomes, which are released by the cells, are also engaged in intercellular transmission of chemical signals. These structures are carriers of mRNA, miRNA and proteins. Among the last, VEGF, PDGF, metalloproteases, integrin $\beta 1$, chemokine receptors [35] and $G$ protein coupling receptors [21] are indicated.

In microvesicle transport, no cell-cell contact is required. This contact is necessary to form gap junctions, the way of transporting of low molecular substances and the second messengers, including calcium ions [76].

Direct interaction between the donor and acceptor cells is also characteristic for immunological synapses [55]. Cell-cell contact connected by immunological synapse is probably one of the mechanisms of trogocytosis. In this phenomenon, the acceptor cell acquires from the donor cell fragments of the cell membrane with integrated antigen proteins $[13,53]$. In the case of natural killers, favorable transfer of cytotoxic substances to the donor cell was observed $[55,68]$.

Rustom and co-workers [57], were the first who described, the yet unknown type of intercellular connections. With regard to dimensions and morphology, these structures were called membrane nanotubes or tunneling nanotubes (TNTs) [57]. Actin filaments are the internal structural component of the TNTs observed between rat pheochromocytoma cells PC12. Subsequent research on other types of animal cells has shown that microtubules $[9,48]$ and intermediate filaments [71] may also be present inside the nanotubes.

The variety of the cells, which are able to communicate in vitro using nanotubes, is impressive. Among them are cell line HEK293T [57], HUVEC [73], RT4 and T24 [28], QGY-7703 [44], MSTO-211H [38], or recently HeLa [59]. Mac- 
rophages [48], lymphocytes T [4, 66], NK cells [9] and dendritic cells [59] also communicate readily via TNTs. So far, nanotubes have been observed in vivo only between dendritic cells in mouse cornea [11].

Recent reports indicate also that nanotubes are the tool of bacterial communication [15], which suggests that TNTs are characteristic not only for multicellular animal organisms.

A growing number of the cell types, which are able to form TNTs and a variety of transported elements, including organelles, membrane-associated components, or signal molecules $[27,41]$ prove that these connections represent common mechanism of functional intercellular connectivity. Undoubtedly, the breakthrough discoveries in the research of TNTs were the results, which showed the TNTs participation in $\operatorname{HIV}[16,66,31,32]$, prion protein $\operatorname{PrP}[7,20,34]$, and MDR [50]. Therefore, the membrane nanotubes are the object of intense research, which will allow finding out in detail not only the TNT structure and mechanisms of their function, but first of all the factors that regulate TNTs formation. This knowledge is very important for developing new therapies of some diseases.

The aim of this work is the comprehensive presentation of the current level of the knowledge of TNTs biology and their participation in pathogenesis of some diseases. The presented source data results from multidirectional studies, carried out on the basis of a number of methods and techniques available in cell biology.

\section{METHODS OF NANOTUBULAR STUDIES AND VISUALISATION}

A high restriction in nanotube studies is the fact that TNTs are the structures with very small dimensions and with short lifetime. The identification of these connections is mainly based on the morphological criteria. Observations of TNTs in electron transmission [21, 66, 71] and scanning microscope [15, 71, 31, 32, 75], or using phase-contrast and interference microscope $[8,48]$ allowed mostly the study of TNTs structure.

On the other hand, fluorescence studies give information about the structure of TNTs, as well as of their function in intercellular transfer. Therefore, dyes like DiD i DiO, or green fluorescent protein GFP are widely used [8, 31, 32, 67]. They enable a labeling both components moving along nanotube surface and those transported inside TNTs [23, 48, 66]. To visualize of actin filaments as TNTs cytoskeletal constituents, a phalloidin-Alexa fluor 488 is used, whereas anti- $\alpha$-tubulin monoclonal antibody conjugated with phycoerythrin is used to stain microtubules $[44,48,66,75]$.

The ability of nanotubes to transport the organelles can be estimated by using markers of selected cell compartments. For example, LysoTracker selectively accu- 
mulates in lysosomes [20, 57, 78], ERTracker in endoplasmic reticulum and GolgiTracker which allows detection of Golgi apparatus [31]. For a similar purpose, MitoTracker is used to mark mitochondria $[25,48,52]$. Contrary to rodamine, classic marker of this compartment, MitoTracker accumulates firmly, also during cell fixation. Its accumulation does not depend on the potential of mitochondrial membranes, which facilitates observations of mitochondria transport from cell to cell. Anti-EEA1 antibodies are helpful in localization of endosome transport [32].

Recent results show the possibility of practical application in TNTs research of inorganic markers - photostable nanoparticles defined as quantum dots [14, 44, 74]. After internalization, these nanoparticles form aggregates. Most of them are closed in lysosomes or endosomes and in this way they are transported within nanotubes. With the regard to properties, the quantum dots are considered as a convenient tool in intercellular drug transport study [64].

In TNTs studies, both flow cytometry and microscopy analyses with a wide range of markers for structural constituents as well as transported components are used $[4,8,40,78,75]$. Quantitative estimation of intercellular organelle transport after cytochalasin B treatment, as an agent disorganizing F- actin in nanotubes, can be the example of these studies [8]. It is worth noting that over 3-fold reduction in organelle transfer, obtained in flow cytometry analysis was similar to that obtained in microscopy (2, 9-fold transport decrease).

Markers of the lipid rafts, known as the cell membrane regions with high distribution of cholesterol and sphingomyelin, are used in the study of the influence of the cell membrane composition and organization on TNTs formation and stability [37]. An example of new generation raft marker is ostreolysin, isolated from mushroom Oyster mushroom. Ostreolysin differs from other raft markers, since it can interact between two lipids (cholesterol and sphingomyelin), and it does not bind to any lipid in its pure form.

Proteomic analysis of TNTs provides opportunities to find out the quantitative contribution of their proteins. It applies not only to cytoskeleton proteins, but also proteins of organelles localized within nanotubes [31].

By using fluorescence microscopy and modern computer technology, a new method of automatic detection of nanotubes in vitro has been developed [26]. The environments of the cell culture influences the results obtained. It refers, for example, to the distance between cells. Its detection is difficult when the distance is too small. Introducing of standardized environments of the cell culture may be the solution of this issue. The microstructured platform method allows to that. The cells are attached to the substratum in a controlled and complementary pattern (singularization) [1]. Assuming that nanotube formation may be different in normal and pathological conditions, the data obtained with the method of microstructured platforms would be quite important for research of new drugs influencing TNTs formation. 


\section{STRUCTURE OF TNTS}

With regard to structure, nanotubes are a heterogeneous group of intercellular connections. Depending on the cell type that form TNTs, these structures show certain characteristic features (tab. 1). Structural differentiation is associated with cytoskeleton component and the presence or lack of the cell membrane continuity.

TABLE 1. Heterogeneity of TNTs structure identified in various cell types

\begin{tabular}{c|c|c|c}
\hline CELL TYPE & $\begin{array}{c}\text { CYTOSKELETAL } \\
\text { COMPONENTS }\end{array}$ & $\begin{array}{c}\text { MEMBRANE } \\
\text { CONTINUITY }\end{array}$ & REFERENCES \\
\hline $\begin{array}{c}\text { RT4, T24 } \\
\text { type I } \\
\text { type II }\end{array}$ & actin filaments & + & {$[57]$} \\
\hline $\begin{array}{c}\text { actin filaments } \\
\text { retinal epithelium }\end{array}$ & keratin filaments & + & {$[71]$} \\
\hline $\begin{array}{c}\text { macrophages } \\
\text { type I } \\
\text { type II }\end{array}$ & actin filaments & + & {$[75]$} \\
\hline lymphocytes T & actin filaments, microtubules & + & {$[48]$} \\
\hline lymphocytes T & actin filaments & - & {$[66]$} \\
\hline NK & actin filaments, microtubules & + & {$[4]$} \\
\hline
\end{tabular}

The connections between PC12 cells, described by Rustom and co-workers [57], are the structures with the diameter of $0,05-0,2$ nanometers and the length exceeding several hundred micrometers, and containing actin filaments. Continuity of the cell membrane between PC12 cells provides the cytoplasm communication. This type of TNTs is defined as the open-ended, and it allows the movement of the cell membrane -associated components, as well as of components or organelles (fig. 1).

Nanotubes formed by macrophages are open-ended structures. Among them, two types are distinguished, differing in cytoskeleton component and in diameter [48]. Nanotubes of the type I are thin, with the diameter $<0,7$ micrometer, containing F-actin. Nanotubes belonging to the type II are thick, with the diameter $\geq 0$, 7 micrometer. This type of connections contains F-actin, as well as microtubules. TNTs of lymphocytes T, described by Sowinski and co-workers [66], include only actin filaments and they do not provide the continuity of cell cytoplasm. Hence, 
these nanotubes are defined as close-ended structures. For this type of connections, the transport of components associated with the cell membrane is typical (fig.1).

The reports of Arkwright and co-workers [4] indicate that lymphocytes T stimulated by FasL may also form open-ended TNTs (tab. 1). It suggests that the same type of cells is able to create nanotubes with different structure, depending on coming signals.

Observations on RT4 or T24 cells show that these cells may form short, containing F-actin, connections, with the length of about 30 micrometers, as well as long nanotubes ( $>100$ micrometers), stabilized by keratin filaments [71]. In both cases, they are open-ended nanotubes.

Despite the differences in structure, a lack of physical contact with the substratum is characteristic for all types of TNTs. It allows distinguishing them from similar structures, e. g. filopodia, cytonemes or filopodial bridges. Like TNTs, filopodia contain actin filaments, their average length, however, is only of a few micrometers $[2,8,36,41]$. Cytonemes are actin-rich structures, with the length over one hundred micrometers. They are described as connections involved in morphogen transport in Drosophila melanogaster [33]. In mammals, thin extensions, similar to cytonemes, are called filopodial bridges. With regard to their participation in retroviruses transmission they are also defined as virus cytonemes [61].

Besides, TNTs differ from recently described tubular connections between bronchial epithelium cells, although both types of structures are not attached to the substratum, and both contain actin and microtubules [79]. Epithelial bridges are wider (1-20 micrometers) and longer (25 micrometer -1 millimeter) than nanotubes. They are stable for more than 48 hours, whereas for TNTs this time is no longer than a few hours [79].

a.

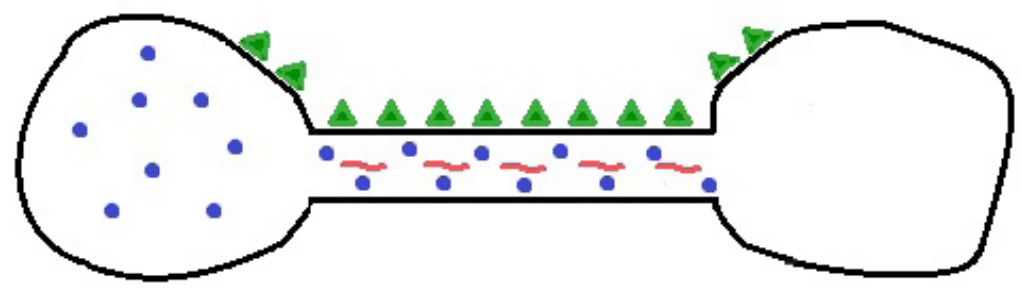

b.

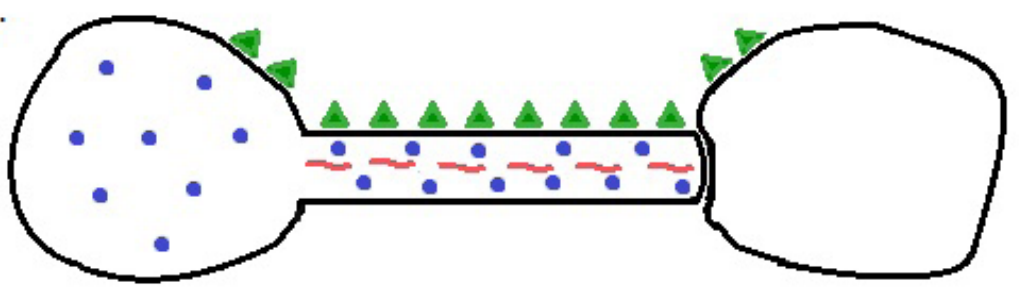

FIGURE 1. Scheme of TNTs structure a) open-ended, b) close-ended 


\section{MECHANISMS OF TNTS FORMATION}

Until now, two mechanisms of TNTs formation have been known. One of them is the mechanism based on cell ability to form nanotubes from filopodia-like protrusions, containing F-actin. This mechanism of de novo formation of TNTs was described for PC12 cells, among others [8, 57]. During TNTs formation and elongation, polymerization of actin appears. It has been found that this process is inhibited when latrunculin is used as an agent that induces actin depolymerization [57]. Interestingly, cytochalasin B inhibits formation of TNTs, but its influence on already existing TNTs is weak.

Molecular mechanism of actin polymerization during de novo TNTs formation has not yet been fully elucidated. Its similarity to the mechanism of filopodia formation could not be excluded [2]. Perhaps, similar to that process, activation of CDC42 protein, which belongs to Rho protein family, involved in organization of actin microfilaments, occurs [54].

After elongation, the end of protrusion interacts with adjacent cell. In PC12 cells, forming open-ended TNT, an adhesion and membrane fusion occurs. The model proposed by Lokar and co-workers [36] assumes that adhesion by several anchoring junctions within the target cell membrane is required to complete stabilization. In these regions, adhesion proteins like $\mathrm{N}$-cadherin and $\beta$-catenin are concentrated.

The second mechanism of TNTs formation is connected with prior cell-cell contact, after which the cells are separated and a nanotube is formed between them. This type of TNTs formation is typical for cells of immune system, like macrophages [48], or lymphocytes $T$ [66]. The process is dependent on cell-cell contact duration [67]. A short, transient contact between lymphocytes T, lasting about 2-3 minutes, rarely leads to TNTs formation. With increasing duration of cell-cell contact, lasting over a few minutes, an incidence of TNTs formation is greater.

NK cells close contact with other cells has been found to allow formation of immunological synapse at the interface of nanotube with the membrane of the target cell. The synapse mediates in NK cells cytotoxicity [9]. Cell polarization, as well as cell adhesion is necessary to form a mature immunological synapse. The changes within the cell membrane and F-actin rearrangement play a key role in this process [19].

The cell membrane organization has a significant effect on TNTs formation and their stability. From the study of Lokar and co-workers [37], it has been found that the presence of cholesterol is very important. Its partial depletion from the cell membrane under methyl- $\beta$-cyclodextrin, as well as under cell culture medium withe out cholesterol, induces marked reduction of TNTs formation.

The increase in TNTs number of RT4 and T24 cells was observed with the increase of the number of ganglioside molecules GM1 within this area of the cell membrane [28]. This agent is a known lipid raft marker, which binds cholera toxin $B$ [63]. It was shown that the number of TNTs in tumour cells T24 is indeed larger 
than in normal RT4 cells. The difference was observed in standard cell culture, as well as in lower temperature and after cholera toxin B treatment. More stimulated effect of the toxin on tumour cells may be the result of higher lipid raft content within the cell membranes [28].

Studies on artificial lipid systems may be also helpful in understanding of mechanisms and forces that rule the process of TNTs formation. The formation of nanotubular connections between liposomes was described in literature [71]. In contrast to intercellular nanotubes, these connections, exhibit a mechanic stability, despite that they do not have actin filaments as strengthening elements. Lipid rafts, rich in cholesterol and sphingomyelin, with a suitable curvature, may be responsible for stability of nanotubes in artificial and biological systems [30,71]. In the area where a nanotube is formed, a tubular-shaped, so called anizothrope curvature, is preferred. I-BAR proteins, associated with lipid rafts, are additional factor influencing on the cell membrane geometry. Their role in interactions between actin filaments and the cell membrane is known [80]. TNTs stability is guaranteed only over a specific range of the cell membrane curvature, when the I-BAR proteins density is not high [29].

\section{REGULATORY FACTORS OF TNTS FORMATION}

The stress induced by hypoxia is a factor that stimulates cells to form TNTs de novo. The research conducted on astrocytes and neurons from rat hippocampus revealed that these cells are stimulated to form nanotubes in the presence of $\mathrm{H}_{2} \mathrm{O}_{2}$ [73]. Cells undergoing stress begin to form nanotubes into unstressed cells. The very same authors showed also that in hypoxia a protein p53 is activated, which is a starting point for a cascade of events leading finally to TNTs formation (fig.2). The epidermal growth factor gene is one of those which are activated by $\mathrm{p} 53$ protein. As a result, Akt/ PI3K/mTOR signaling pathway is turned on [73]. The results of Lou and co-workers [2012] suggest the important role of mTOR in the TNTs formation. The process is stopped in the presence of mTOR inhibitor (Everolimus). Stimulation of nanotube formation in the glucose-rich and low $\mathrm{pH}$ medium may be the effect of mTOR pathway hyperactivation [38].

$\mathrm{M}-\mathrm{Sec}$ protein is another regulatory factor of TNTs formation [22, 47]. Its expression is increased in hypoxia and may be regulated by p53 protein. [73]. $\mathrm{M}-\mathrm{Sec}$, induced by TNF- $\alpha$, is defined as a one of possible molecular markers of TNTs connections [47]. The results of research on M-Sec role in TNTs formation indicate that this protein cooperates with RaIA GTPase. RaIA is involved in rearrangement of F-actin in cell and in the vesicular transport, which is responsible for delivery of relevant membrane components in the region of nanotube formation. $\mathrm{M}-\mathrm{Sec}$ and RaIA regulate actin polimerization, including in it CDC42 protein, which was mentioned earlier. 


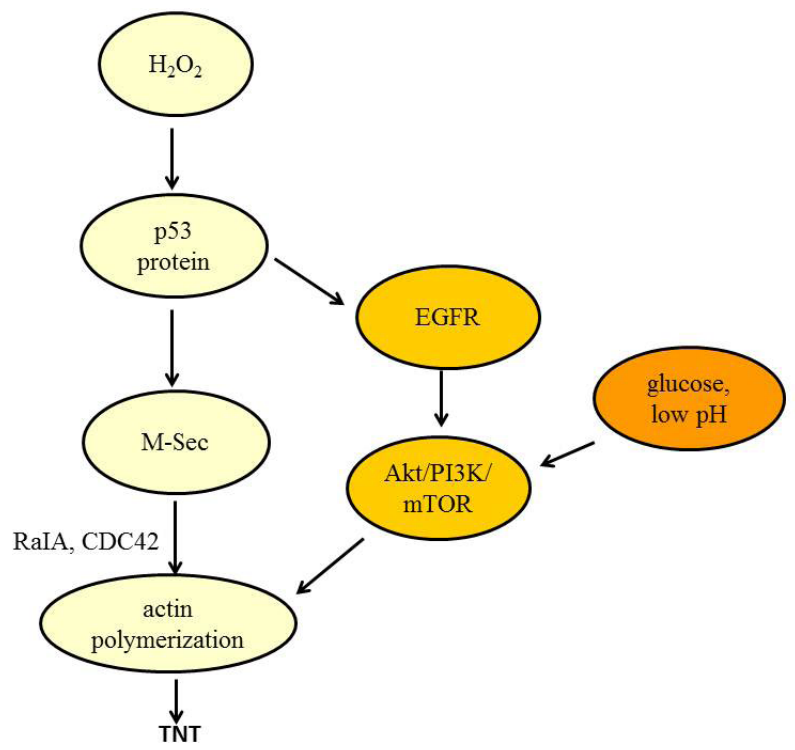

FIGURE 2. Factors stimulating TNTs formation

According to the data shown above, the M-Sec protein participation as a regulatory factor in TNTs formation is probably limited to the nanotubes with actin filaments. It suggests that the formation of TNTs, containing microtubules, needs other regulatory factors than M-Sec protein [33]. Studying them as well as carrying further studies on M-Sec role seems to be quite important. This may lead to the discovery of some drugs affecting the factor activity, and in this way regulating the TNTs formation process.

\section{MECHANISMS OF NANOTUBULAR TRANSPORT AND ITS PHYSIOLOGICAL ROLE}

According to the numerous studies, membrane nanotubes are intercellular transport routes of cytosol components, as well as organelles, and components associated with the cell membrane (tab. 2). Open-ended nanotubes, containing only F-actin allow unidirectional transport [16, 20, 57], whereas the nanotubes with F-actin, as well as microtubules, are capable of bidirectional transport [44, 48].

Myosin is involved in the transport along actin filaments [44, 74], kinesin and dynein are protein motors associated with microtubule transport [44]. This transport is an active process, which is blocked by azide, an agent that inhibits ATP synthesis $[48,74]$. 
When the diameter of cargo is greater than the inner diameter of the nanotube, the TNTs transport can take place via dilatations. The distentions along nanotubes resemble gondolas $[14,71]$. These structures form an integral part of TNTs connections and they include both F-actin and keratin filaments. [71]. An average speed of movement within the gondolas is 40 nanometers/second.

\section{ORGANELLE TRANSPORT AS A CELLULAR RESCUE STRATEGY}

Organelle transport via nanotubes seems to play an important role especially when cells are damaged, or undergo premature senescence. Hence, its importance is emphasized in repair cell strategy.

Diabetes, atherosclerosis and chronic kidney inflammation are accompanied by oxidative stress result in stress-induced premature senescence of vascular endothelium [18]. Lysosomal dysfunction, ganglioside cell accumulation and autophagy are the effects of stressed conditions [51]. Transport of lysosomes with integral membrane was observed in nanotubes directed from intact endothelial progenitor cells to HUVEC cells exposed to an agent that induces collagen I glication. An average speed of this process was 1 micrometer/minute [77].

A repair effect such as increased transport of mitochondria was observed between normal EPC cells and HUVEC cells treated with cytotoxic concentration of adriamycin (doxorubicin) and showing the mitochondria dysfunction [77].

The results of Cselenyak and co-workers [12] are another example of TNTs contribution in rescue cell strategy. Viability of rat cardiomyoblasts incubated for 150 minutes in the glucose-free medium, and in the presence of $0,5 \% \mathrm{O}_{2}$ and $99,5 \% \mathrm{~N}_{2}$ was increased significantly after the contact with the normal mouse mesenchymal stem cells and the cytosolic contents as well as mitochondria transport.

The results of Plotnikov and co-workers [52] show that TNTs can also mediate in differentiation of human mesenchymal multipotent stromal cells MMSC. Mitochondrial transport from differentiated renal tubular cells RTC is accompanied by cytosolic components responsible for MMSC differentiation.

An open question still remains of how the formation of nanotubes and the selective transport in non-stressed cells is initiated. Probably, the non-stressed cells send signals to the intact cells and these start to form nanotubes. An accumulation of phosphatidylserine in outer monolayer of the cell membrane can be such a signal [78]. A binding of phosphatidylserine by annexin $V$ blocks the signal and reduces organelle transport significantly. 


\section{CALCIUM SIGNAL TRANSMISSION}

Watkins and Salter, first showed the contribution of TNTs in $\mathrm{Ca}^{2+}$ transmission [27]. Calcium signal via nanotubes is passed between immunological cells in a matter of only a few seconds from a distance of even several hundred of micrometers. Further studies revealed that this transmission is possible also in retinal pigment epithelial cells, HEK cells, or neuroblastoma cells SH-SY5Y [22, 65, 72, 75]. In close-ended TNTs, a depolarization is directed from an activated cell to a non-activated cell $[72,75]$. This causes transient increase in $\mathrm{Ca}^{2+}$ concentration, as a result of potential-dependent calcium channels activation.

A protein component of gap junctions, connexin 43, was found at the interface between nanotube and the surface of the target cell $[72,75]$. The fact that gap junctions blocker, a meclofenamic acid, abolishes depolarization transmission in the target cell and opening of voltage-dependent calcium channels, indicates the important role of these connections in $\mathrm{Ca}^{2+}$ signal transfer.

It was found, that a direct flow of calcium ions via TNTs may also follow. However, open-ended nanotubes are involved in this process [22, 65]. At the same time, it was demonstrated, that a passive flow of $\mathrm{Ca}^{2+}$ ions is not sufficient for an effective activation of the target cell [65]. Inositol triphosphate receptors are engaged in the signal amplification. They were found within endoplasmic reticulum inside TNTs.

\section{APOPTOTIC TNTS DEATH SIGNAL TRANSMISSION}

Recent studies have shown that activation of lymphocytes T by FasL, which is involved in extrinsic apoptotic death pathway, stimulates cells to form nanotubes and to exchange of death signal $[4,40]$. Mutation in the cytoplasmic domain of the Fas receptor, characteristic for the autoimmune lymphoproliferative syndrome ALPS, excludes the possibility of lymphocytes T stimulation to form TNTS connections [4].

The death signal distribution may be the result of membrane-associated FasL ligand movement along the nanotubes [4]. The mobility of caspase-3, one of the enzymes responsible for apoptosis realization, was also demonstrated within the nanotubes [4]. The movement of membrane-associated FasL can be explained by the close-ended type of nanotubes [66], whereas the transport of caspase-3 between the cells connected is possible only with participation of the open-ended TNTs [4]. 


\section{TRANSPORT OF THE CELL MEMBRANE-ASSOCIATED COMPONENTS}

The transport of the cell membrane-associated components is a particular type of transport occurring with the TNTs participation. It includes movement of GPI-anchored proteins, antigen proteins, or membrane receptors (tab. 2).

TABLE 2. Types of cargo transported by TNTs

\begin{tabular}{c|c|c}
\hline CATEGORY & COMPONENTS & REFERENCES \\
\hline \multirow{2}{*}{ organelles } & Golgi, ER & {$[31]$} \\
& endosomes & {$[32]$} \\
& lysosomes & {$[20,57,78]$} \\
cytosol & mitochondria & {$[25,48,52]$} \\
\hline \multirow{2}{*}{ calcium signal } & calcein & {$[52]$} \\
& cytosol GFP & {$[23]$} \\
\hline \multirow{2}{*}{ apoptotic death signal } & Ca ${ }^{2+}$ & {$[72]$} \\
& Ca ${ }^{2+}$, inositol triphosphate receptor & {$[65]$} \\
\hline \multirow{2}{*}{ cell membrane } & caspase-3 & {$[4]$} \\
& cytotoxic (perforins) & {$[6]$} \\
\hline
\end{tabular}

Recent reports of Schiller and co-workers [59] indicate that TNTs are responsible for the transport of MHC class molecules between HeLa cells. The degree of this transport is significantly reduced in the presence of latrunculin A, inhibitor of actin polymerization. It was found that GFP-labeled antigen protein HLA-A2 may be transported not only as a membrane-associated component, but also through the cytoplasm. 
The results of Takahashi and co-workers [70], that suggest TNTs participation in the transport of transmembrane protein DC-STAMP, should also be noted. This protein plays a key role in the fusion of osteoclast progenitors in osteoclastogenesis.

The accumulation of the second messenger cAMP in the target cell occurs as a result of TNTs mediated transport of membrane receptors GPCR [21]. It suggests that after transport these receptors remain functionally competent in the target cell.

The difference in membrane potential, presented across the lipid bilayer, is probably the membrane flow for the TNTs transport associated with the cell membrane, directing cargo from the regions with low potential to the region with the higher one [27].

\section{TNTS PARTICIPATION IN PATHOMECHANISM OF DISEASES}

Existing data suggest that membrane nanotubes may play the important role in the development of some diseases. HIV, as well as $\mathrm{PrP}^{\mathrm{sc}}$ protein may be transported by nanotubes from infected cells to non-infected cells $[16,20,34,66]$.

The intercellular spread of bacteria can also occur through the nanotubes [48].

The important issue is the TNTs contribution towards pathogenesis and invasion of cancer. It is suggested that nanotubes may participate in the acquisition of the chemotherapy resistance by tumour cells and in organelle transfer, including mitochondria transfer $[38,39,50]$. This in turn can be an important strategy to ensure effective respiration by tumour cells [38].

\section{HIV TRANSFER}

Transmission of a pathogen such as HIV is several thousand times more efficient in a case of physical cell-cell contact rather than during an uptake of cellfree virus $[10,66]$. The viral propagates preferentially across polysynapses, when cell-cell contact is possible. These structures are formed between infected lymphocyte $\mathrm{T}$ and a few non-infected ones [56].

The results presented by Sowinski $[66,67]$ indicate that TNTs are also involved in HIV transmission. These structures are classified as close-ended nanotubes. They differ significantly from viral cytonemes, which participate in both HIV and MLV propagation [61, 62, 67].

An important difference between virus cytonemes and TNTs is the dependence of the mechanism of their formation on interactions between virus envelope protein and CD4 antigen in the target cell membrane (tab. 3). The process of the virus transport relies on its surfing along outer surface of cytonemes. 
TABLE 3. Characteristics of close-ended TNTs and viral cytonemes, involved in HIV transfer. According to [67] changed

\begin{tabular}{|c|c|c|}
\hline FEATURE & CLOSE-ENDED TNTS ${ }^{\mathrm{A}}$ & VIRUS CYTONEMES ${ }^{\mathrm{B}}$ \\
\hline morphology and formation & $\begin{array}{l}\text { straight, above the substratum, } \\
\text { formed after intercellular contact }\end{array}$ & $\begin{array}{l}\text { curved, adherent to substratum, } \\
\text { formed by non-infected cells, } \\
\text { through de novo mechanism }\end{array}$ \\
\hline length & 25 micrometers & 5,8 micrometers \\
\hline diameter & $180-380$ nanometers & $75-200$ nanometers \\
\hline HIV transfer mechanism & $\begin{array}{c}\text { independent of interactions } \\
\text { between virus envelope protein } \\
\text { and CD4 transfer of GFP-labeled } \\
\text { capsid protein from infected to } \\
\text { non-infected cell }\end{array}$ & $\begin{array}{l}\text { dependent on interactions between } \\
\text { virus envelope protein and CD } 4 \\
\text { transfer along the outer surface of } \\
\text { cytonemes }\end{array}$ \\
\hline speed of transfer & $0,08 \pm 0,03$ micrometers $/$ second & $0,011 \pm 0,003$ micrometers/second \\
\hline
\end{tabular}

a- results from [66]

$\mathrm{b}-$ results from $[61]$

The formation of nanotubes, involved in HIV transfer, is not dependent on interactions between virus envelope protein and CD4 antigen on the target cell. A capsid protein transfer of GFP-labeled HIV passes along nanotubes. It is unknown, the process is a transfer inside nanotubes or on a surface of TNTs. How virus transported via nanotubes overcomes a barrier of the cell membrane is also still unclear. Probably, receptor-dependent endocytosis takes place at the end of TNTs [66].

Recent results of Kadiu and Gendelman [31,32] suggest that in the virus propagation open-ended TNTs are involved. HIV infection stimulates formation of the connections with the length about one hundred micrometers and containing both actin filaments and microtubules. Fluorescent labeled HIV envelope and capsid proteins have been find in endosomes and Golgi apparatus.

Detailed proteomic analyses of isolated TNTs have shown that HIV proteins were $3 \%$ of the overall identified proteins. The distribution of other proteins was as follows: cytosolic (23\%), cytoskeletal (19\%), cell membrane (15\%), endosome (14\%), mitochondrial (7\%), ER (9\%), Golgi (4\%). Transfer of sequestered virus within TNTs connections is a total protection against the extracellular environment, in particular against the immune system. 


\section{TNTS IN NEURODEGENERATIVE AND PRION DISEASES}

It is noted that TNTs are able to transport of damaged mitochondria between the cells of the central nervous system. This transfer may contribute to the development of neurodegenerative diseases, like Alzheimer or Parkinson [43]. $\alpha$-synuklein is probably the damaged factor of mitochondria, leading to degradation and fragmentation of these organelles [45]. Mitochondria failure induced by reactive oxygen species participate in neurons degeneration in patients with Alzheimer [69].

It is believed that nanotubes are the tool for spreading both exogenous and endogenous $\mathrm{PrP}^{\mathrm{sc}}$ protein, which is responsible for Creutzfeldt-Jakob disease [7, 20, 34].

Until now, it has been assumed that prion protein may be transfered by a direct cell-cell contact, with exosomes participation or by secretion of $\mathrm{PrP}^{\mathrm{sc}}$ from infected cell to the extracellular space [3]. TNTs formed between dendritic cells and neurons as well as between neurons alone play a crucial role in transfer of exogenous $\mathrm{PrP}^{\mathrm{sc}}$ from digestive tract to perihperal and then to the central nervous system [20,34].

The mechanism of PrPsc transfer and the type of TNTs participating in this process is not yet completely known. Neither transport within endosomes, nor the one along nanotube surface can be excluded [41]. According to data by Langevin and co-workers [34], the process occurs as a transfer of $\mathrm{PrP}^{\mathrm{sc}}$ aggregates (aggregosomes) from cytoplasm of infected to non-infected cell. It suggests the contribution of open-ended nanotubes.

\section{TRANSFER OF BACTERIA AND THEIR RESISTANCE TO ANTIBIOTICS}

Onfelt and co-workers' results [48] show, that bacteria Mycobacterium bovis bacillus, with GFP expression, can surf between human macrophages, using nanotubes with the diameter $<0,7$ micrometer, and containing F-actin. The first step of this process is bacteria binding to nanotube membrane, then cell surfing along nanotube surface and the next step is phagocytosis in the place, where the nanotube has a contact with target cell. Bacteria bind also to the nanotubes with higher diameter, containing microtubules in addition to actin. However, no surfing in the direction of the target cell is observed [48], which indicates the selectivity of this process.

Bacteria cells, able to form nanotube connections [15], can use them for nongenetic and genetic transfer of their resistance to antibiotics. Protein and plasmid transport was found not only between bacteria of the same species Bacillus subtilis. The transport is possible also between different species Bacillus subtilis and Staphylococcus aureus, or Bacillus subtilis and Escherichia coli [15]. 


\section{TNTS IN TUMOUR DISEASE}

In some conditions, the response of tumour cells to the factors regulating TNTs formation is stronger than the response of normal cells [28]. A low $\mathrm{pH}$ is one of the stimulating factors, and the connections formed allow, among others, mitochondria transport [38]. According to Lou and co-workers [38], tumour cells, paradoxically to the Warburg effect, supporting mainly anaerobic metabolism, share of mitochondria, which may provide increase in energy production and thereby cell proliferation.

TNTs participate also in transfer of mitochondrial DNA and its mutations [24]. It was found that cells from the individual tumour patient show a high heterogeneity of mitochondrial DNA because of mutations [24].

Nanotubes are probably a route to acquire the resistance to chemotherapy in tumour cells [50]. So far, this phenomenon has been defined as multi-drug resistance connected with an overexpression and high activity of the cell membrane proteins, belonging to the $\mathrm{ABC}$ family [60]. As a result, active efflux of drugs from cell occurs, which makes it impossible to reach therapeutic drug concentrations. Among proteins responsible for this, a main attention is paid to MDR protein. MDR is a protein with molecular mass of about $170 \mathrm{kD}$, present in epithelial cells of intestine, kidney and in blood-brain barrier [60].

In tumours developing from tissues with high expression of MDR gene, a low response to chemotherapy is observed. For other tumours, exposure to cytotoxics may lead to a de novo expression of MDR in cells, which did not show the resistance to chemotherapy. [49]. Such tumours, become secondarily multidrug resistant.

The results of Pasquier and co-workers [50], obtained on the MCF-7 cell line, have shown that nanotubes participate in the MDR protein transfer. It means that tumour cells, lacking the expression of MDR (MDR') and sensitive to drugs, may acquire the resistance as a result of MDR transfer from $\mathrm{MDR}^{+}$cells.

\section{SUMMARY}

Actually, the membrane nanotubes are defined as a very important form of intercellular communication. It refers to animal, as well as bacterial cells. As a result of intensively conducted studies, our knowledge about the structure and the role of TNTs is becoming more comprehensive. Undoubtedly, the development of the methods, which allow visualization of the structure, as well as the type of intercellular transport taking place with the participation of TNTs, contributed to this. These structures are involved in many physiological processes, including immunological response and cell repair. The main attention is paid to their heterogeneity and thus raises the question of which factors determine the type of TNTs 
formed by cells, and whether their formation is a cell-specific process. Some data suggest that depending on the signals, cells may form different types of membrane nanotubes. Taking into account the fact that these connections are the tool for intercellular communication also in pathological conditions, it is quite important to know the exact mechanism of their formation and the factors that regulate this process. Understanding of these issues may lead to development of drugs and strategy reducing the spread of certain diseases in the human body.

\section{REFERENCES}

[1] Abel M, Riese SR, Schlicker O, Bukoreshtliev N, Gerdes H, Spatz JP, Rustom A. Microstructured platforms to study nanotube-mediated long-distance cell-to-cell connections. Biointerphases 2011; 6: $22-31$.

[2] Aвоunit S, Zurzolo $\mathrm{CH}$. Wiring through tunneling nanotubes -from electrical signals to organelle transfer. J Cell Sci 2012; 125: 1089-1098.

[3] Aguzzi A., Calella AM. Prions: Protein aggregation and infectious diseases. Physiol Rev 2009; 89: 1105-1152.

[4] Arkwright PD, Luchetti F, Tour J, Roberts CH, Ayub R, Morales AP, Rodriguez J, Gilmore A, CANONICO B, PAPA S, EsPosti MD. Fas stimulation of T lymphocytes promotes rapid intercellular exchange of death signals via membrane nanotubes. Cell Res 2010; 20:72-88.

[5] Baluska F, Hlavacka A, Volkmann D, Menzel D. Getting connected: actin-based cell-to-cell channels in plants and animals. Trends Cell Biol 2004; 14: 404-408.

[6] Belting M, Wittrup A. Nanotubes, exosomes, and nucleic acid-binding peptides provide novel mechanisms of intercellular communication in eukaryotic cells: implications in health and disease. J Cell Biol 2008; 183: 1187-1191.

[7] Brundin P, Melki R, Kopito R. Prion-like transmission of protein aggregates in neurodegenerative diseases. Mol Cell. Biol 2010: 11: 301-307.

[8] Bukoreshtliev NV, Wang X, Hodneland E, Gurke S, Barroso J, Gerde HH. Selective block of tunneling nanotube (TNTS) formation inhibits intercellular organelle transfer between PC12 cells. FEBS Lett 2009; 583: 1481-1488.

[9] Chauveau A, Aucher A, Eissmann PH, Vivier E, Davis D. Membrane nanotubes facilitate long-distance interactions between natural killer cells and target cells. PNAS 2010; 107: 5545-5550.

[10] Chen P, Hubner W, Spinelli MA, Chen BK. Predominant mode of human immunodeficiency virus transfer between $\mathrm{T}$ cells is mediated by sustained Env-dependent neutralization-resistant virological synapses. J Virol 2007; 81: 12582-12595.

[11] Chinnery HR, Pearlman E, Mcmenamin PG. Cutting edge: Membrane nanotubes in vivo: a feature of MHC Class II+ cells in the mouse cornea. J Immunol 2008; 180: 5779-5783.

[12] Cselenyak A, Pankkotai E, Horvath E, Kiss L, Lacza Z. Mesenchymal stem cells rescue cardiomyoblasts from cell death in an in vitro ischemia model via direct cell-to-cell connections. BMC Cell Biol 2010; 11: 29.

[13] Daubeuf S, Aucher A, Bordier Ch, Salles A, Serre L, Gaibelet G, Faye J-Ch, Favre G, Joly E, HUDRISIER D. Preferential transfer of certain plasma membrane proteins onto $\mathrm{T}$ and $\mathrm{B}$ cells by trogocytosis. PLoS ONE 2010; 5: e8716.

[14] Domhan S, Ma L, Tai A, Anaya Z, Beheshti A, Zeier M, Hlatky L, Abdollah I. Intercellular communication by exchange of cytoplasmic material via tunneling nanotube like structures in primary human renal epithelial cells. PLOS ONE 2011; 6: e21283. 
[15] Dubey GP, Ben-Yenuda S. Intercellular nanotubes mediate bacterial communication. Cell 2011; 144: 590-600.

[16] Eugenin EA, Gaskill PJ, Berman JW. Tunneling nanotubes (TNTS) are induced by HIV-infection of macrophages: a potential mechanism for intercellular HIV trafficking. Cell Immunol 2009; 254: $142-148$.

[17] Gerdes HH, Bukoreshtliev NV, Barroso JF. Tunneling nanotubes: A new route for the exchange of components between animal cells. FEBS Lett 2007; 581: 2194-2201.

[18] Goligorsky MS, Chen J, Patschan S. Stress-induced premature senescence of endothelial cells - a perilous state between recovery and point of no-return. Curr Opin Hematol 2009; 16: 215-219.

[19] Gordon-Alonso M, Veiga E, Sanchez-Madrid F. Actin dynamics at the immunological synapse. Cell Health Cytoskel 2010; 2: 33-47.

[20] Gousset K, Schiff E, Langevin C, Marijanovic Z, Caputo A, Browman DT, Chenouard N, De Chaumont F, Martino A, Enninga J, Olivo-Marin JC, Mannel D, Zurzolo C. Prions hijack tunneling nanotubes for intercellular spread. Nat Cell Biol 2009; 11: 328-336.

[21] Guescini M, Leo G, Genedani S, Carone S, Pederzoli F, Ciruela F, Guidolin D, Stocchi V, Mantuano M, Borroto-Escuela Do, Fuxe K, Agnati LF. Microvesicle and tunneling nanotube mediated intercellular transfer of g-protein coupled receptors in cell cultures. Exp Cell Res 2012; 318: 603-613.

[22] Hase K, Kimura S, Takatsu H, Ohmae M, Kawano S, Kitamura H, Ito M, Watarai H, Hazelett Cc, Yeaman $\mathrm{CH}$, OhNo H. M-Sec promotes membrane nanotube formation by interacting with RaI and the exocyst complex. Nat Cell Biol 2009; 11: 1427-1432.

[23] He K, Luo W, Zhang Y, Liu F, Liu D, Xu L, Qin L, Xiong C, Lu Z, Fang X, Zhang Y. Intercellular transportation of quantum dots mediated by membrane nanotubes. ACS Nano 2010; 4: 3015-3022.

[24] He Y, Wu J, Dressman DC, Iacobuzio-Donahue CH, Markowitz SD, Velculescu Ve, Diaz La, Kinzler KW, Vogelstein B, Papadopoulos N. Heteroplasmic mitochondrial DNA mutations in normal and tumor cells. Nature 2010; 464: 610-614.

[25] He K, Zhang X, Dang S, Ma X, Liu F, Xu M, Lv Z, Han D, Fang X, Zhang Y. Long-distance intercellular connectivity between cardiomyocytes and cardiofibroblasts mediated by membrane nanotubes. Cardiovasc Res 2011; 92: 39-47.

[26] Hodneland E, Lundervold A, Gurke S, Tai XCh, Rustom A, Gerdes HH. Automated detection of tunneling nanotubes in 3D images. Cytom Part A 2006; 69A: 961-972.

[27] Hurtig J, Chiu DT, Onfelt B. Intercellular nanotubes: insights from imaging studies and beyond. Wiley Interdiscip Rev Nanomed Nanobiotech 2010; 2: 260-276.

[28] Kabaso D, Lokar M, Kralj-Iglic V, Veranic P, Iglic A. Temperature and cholera toxin B are factors that influence formation of membrane nanotubues in RT4 and T24 urothelial cancer cell lines. Int $J$ NanoMedicine 2011; 6:495-509.

[29] Kabaso D, Bobrovska N, Góźdź W, Gov N, Kralj-Iglic V, Veranic P, Iglic A. On the role of membrane anisotropy and BAR proteins in the stability of tubular membrane structures. J Biomech 2012; 45: 231-238.

[30] Kabaso D, Bobrovska N, Góźdź W, Gongadze E, Kralu-Iglic V, Zorec R, Iglic A. The transport along membrane nanotubes driven by the spontaneous curvature of membrane components. Bioelectrochemistry 2012; 87: 204-210.

[31] Kadiu I, Gendelman HE. Macrophage bridging conduit trafficking of HIV-1 through the endoplasmic reticulum and Golgi network. J Proteome Res 2011; 10: 3225-3238.

[32] Kadiu I, Gendelman HE. Human Immunodeficiency virus type 1 endocytic trafficking through macrophage bridging conduits facilitates spread of infection. J Neuroimmune Pharmacol 2011; 6: 658-675.

[33] Kimura S, Hase K, Ohno H. Tunnel ing nanot ubes: Emerging view of their molecular components and formation mechanisms. Exp Cell Res 2012; 318: 1699-1706.

[34] Langevin CH, Gousset K, Costanzo M, Richard-Le Goff O, Zurzolo C. Characterization of the role of dendritic cells in prion transfer to primary neurons. Biochem J 2010; 431: 189-198. 
[35] Lee Th, D`Asti E, Magnus N, Al-Nedawi K, Meehan B, Rak J . Microvesicles as mediators of intercellular communication in cancer -the emerging sciene of cellular "debris". Semin Immunopathol 2011; 33:455-467.

[36] Lokar M, Iglic A, Veranic P. Protruding membrane nanotubes: attachment of tubular protrusions to adjacent cells by several anchoring junctions. Protoplasma 2010; 246: 81-87.

[37] Lokar M, Kabaso D, Resnik N, Sepcic K, Kralu-Iglic V, Veranic P, Zorec R, Iglic A. The role of cholesterol-sphingomyelin membrane nanodomains in the stability of intercellular membrane nanotubes. Int J Nanomed 2012; 7: 1891-1902.

[38] Lou E, Fujisama S, Morozov A, Barlas A, Romin Y, Dogan Y, Gholami S, Moreira A, ManoVA-Todorova K, Moore MA. Tunneling nanotubes provide a unique conduit for intercellular transfer of cellular contents in human malignant pleural mesothelioma. PLOS ONE 2012; 7: e33093.

[39] Lou E, Fujisana S, Barlos A, Romin Y, Manova-Todorova K, Moore MA, Subramanian S. Tunneling nanotubes: A new paradigm for studying intercellular communication and therapeutics in cancer. Commun Integr Biol 2012; 5: 399-403.

[40] Luchetti F, Canonico B, Arcangeletti M, Guescini M, Cesarini E, Stocchi V, Degli Esposti M, Papa S. Fas signaling promotes intercellular communication in T cells. PLOS ONE 2012; 7: e35766.

[41] Marzo L, Gousset K, Zurzolo Сн. Multifaceted roles of tunneling nanotubes in intercellular commum nication. Front Physiol 2012; 3: 1-14.

[42] Mathivanan S, Ji H, Simpson RJ. Exosomes: Extracellular organelles important in intercellular communication. J Proteomics 2010; 73: 1907-1920.

[43] Mcgowan M. Tunnel ing nanot ubes -crossing the bridge. J Cell Mol Biol 2011; 9: 11-18.

[44] Mi L, Xiong R, Zhang Y, Li Z, Yang W, Chen J, Wang P. Microscopic observat ion of the int er cel lular transport of CdTe quantum dot aggregates through tunneling-nanotubes. J Biomat Nanobiotech 2011; 2: $173-180$.

[45] Nakamura K, Nemani VM, Azarbal F, Skibinski G, Levy JM, Egami K, Munishiina L, Zhang J, Gardner B, Wakabayashi J, Sesaki H, Cheng Y, Finkbeiner S, Nussbaum RL, Masliah E, Edwards RH. Direct membrane association drives mitochondrial fission by the Parkinson disease-associated protein $\alpha$-synuclein. J Biol Chem 2011; 286: 20710-20726.

[46] Niu X, Gupta K, Yang Jt, Shamblott MJ, Levchenko A. Physical transfer of membrane and cytoplasmic components as a general mechanism of cell-cell communication. J. Cell Sci 2008; 122: 600-610.

[47] Ohno H, Hase K, Kimura S. Emerging secrets of tunneling nanotube formation. Commum Integr Biol. 2010; 3: 231-233.

[48] Onfelt B, Nedvetzki S, Benninger RK, Purbhoo MA, Sowinski S, Hume AN, Seabra MC, Neil MA, FRENCH PM, DAVIS D. Structurally distinct membrane nanotubes between human macrophages support long-distance vesicular traffic or surfing of bacteria. J Immunol 2006; 177: 8476-8483.

[49] Pasquier J, Magal P, Boulange-Lecomte C, Webb G, Le Foll F. Consequences of cell-to-cell P-glycoprotein transfer on acquired multidrug resistance in breast cancer: a cell population dynamics model. Biol Direct 2011; 6: 1-18.

[50] Pasquier J, Galas L, Boulange-Lecomte C, Rioult D, Bultelle F, Magal P, Webb G, Le Foll F. Different modalities of intercellular membrane exchanges mediate cell-to-cell P-glycoprotein transfers in MCF-7 breast cancer cells. J Biol Chem 2012; 287: 7374-7387.

[51] Patschan S, Chen J, Gealekman O, Krupincza K, Wang M, Shu L, Shayman Ja, Goligorsky MS. Mechanisms of premature cell senescence: lysosomal dysfunction and ganglioside accumulation in endothelial cells. Am J Physiol Renal Physiol 2008; 294: 100-109.

[52] Plotnikov EY, Khryapenkova TG, Galkina SI, Sukhikh GT, Zorov DB. Cytoplasm and organelle transfer between mesenchymal multipotent stromal cells and renal tubular cells in co-culture. Exp Cell Res 2010; 316: 2447-2455.

[53] Rechavi O, Goldstein I, Kloog Y. Intercellular exchange of proteins: The immune cell habit of sharing. FEBS Lett 2009; 583: 1792-1799. 
[54] Ridley AJ. Life at the leading edge. Cell 2011; 145: 1012-1022.

[55] Roda-Navarro P, REYBURn HT. Intercellular protein transfer at the NK cell immune synapse: mechanisms and physiological significance. FASEB J 2007; 21: 1636-1646.

[56] Rudnicka D, Feldmann J, Porrot F, Wietgrefe S, Guadagnini S, Prevost M-Ch, Estaquier J, Haase AT, Sol-Foulon N, Schwartz O. Simultaneous cell-to-cell transmission of Human Immunodeficiency Virus to multiple targets through polysynapses. J Virol 2009; 83: 6234-6246.

[57] Rustom A, Saffrich R, Markovic I, Walther P, Gerdes HH. Nanotubular highways for intercellular organelle transport. Science 2004; 303:1007-1010.

[58] Rustom A. Hen or Egg? Some thoughts on tunneling nanotubes. Nat Genetic Engin Nat Genome 2009; 1178: $129-136$.

[59] Schiller Ch, Huber JE, Diakopoulos KN, Weiss EH. Tunneling nanotubes enable intercellular transfer of MHC class I molecules. Hum Immunol 2013; 74: 412-416.

[60] Sнавом FJ. ABC multidrug transporters: structure, function and role in chemoresistance. Pharmacogenomics 2008; 9: 105-127.

[61] Sherer NM, Lehmann MJ, Jimenez-Soto LF, Horensavitz Ch, Pypaert M, Mothes W. Retroviruses can establish filopodial bridges for efficient cell-to-cell transmission. Nat Cell Biol 2007; 9: 310-315.

[62] Sherer NM, Mothes W. Cytonemes and tunneling nanotubules in cell-cell communication and viral pathogenesis. Trends Cell Biol 2008; 9: 414-420.

[63] Simons K, Sampaio JL. Membrane organization and lipid rafts. Cold Spring Harb Perspect Biol 2011; 3: a004697.

[64] Singh R, NALWA HS. Medical applications of nanoparticles in biological imaging, cell labeling, antimicrobial agents and anticancer nanodrugs. J Biomed Nanotechnol 2011; 7: 489-503.

[65] Smith IF, Shai J, Parker I. Active generation and propagation of $\mathrm{Ca}^{2+}$ signals within tunneling membrane nanotubes. Biophys $J$ 2011; 100: L37-L39.

[66] Sowinski S, Jolly C, Berninghausen O, Purbhoo MA, Chauveau A, Kohler K, Oddos S, Eissmann PH, Brodsky FM, Hopkins C, Onfelt B, Sattentau Q, Davis D. Membrane nanotubes physically connect T cells over long distances presenting a novel route for HIV-1 transmission. Nature Cell Biol 2008; 10: 211-219.

[67] Sowinski S, Alakoskela JM, Jolly C, Davis D. Optimized methods for imaging membrane nanotubes between T cells and trafficking of HIV-1. Methods 2011; 53: 27-33.

[68] Stinchcombe JC, Salio M, Cerundolo V, Pende D, Arico M, Griffiths GM. Centriole polarization to the immunological synapse direct secretion from cytolytic cells of both the innate and adaptive immune systems. BMC Biol 2011; 9: 45-52.

[69] Su B, Wang X, Nunomura A, Moreira PI, Lee HG, Perry G, Smith Ma, Zhu X. Oxidative stress signaling in Alzheimer's disease. Curr Alzheimer Res 2008; 5: 525-532.

[70] Takahashi A, Kukita A, Zhang J, Nomiyama H, Yamaza T, Ayukawa Y, Koyano K, Kukita T. Tunneling nanotube formation is essential for the regulation of osteoclastogenesis. J Cell Biochem 2012 /doi/ 10.1002/jcb.24433.

[71] Veranic P, Lokar M, Schutz Gu, Weghuber J, Wieser S, Hagerstrand H. Different types of cell-tocell connections mediated by nanotubular structures. Biophys $J$ 2008; 95:4416-4425.

[72] Wang X, Veruki ML, Bukoreshtliev NV, Hartveit E, Gerdes HH. Animal cells connected by nanotubes can be electrically coupled through interposed gap-junction channels. PNAS 2010; 107: 17194-17199.

[73] Wang Y, CuI J, Sun X, Zhang Y. Tunneling nanotube development in astrocytes depends on p53 activation. Cell Death Differ 2011; 18: 732-742.

[74] Wang ZG, Liu SL, Tian ZQ, Zhang ZL, Tang HW, Pang DW. Myosin-driven intercellular transportation of wheat germ agglutinin mediated by membrane nanotubes between human lung cancer cells. ACS Nano 2012; 6: 10033-10041.

[75] Wittig D, Wang X, Walter C, Gerdes HH, Funk RH, Roehlecke C. Multi-level communication of human retinal pigment epithelial cells via tunneling nanotubes. PLOS ONE 2012; 7: e33195. 
[76] Yao J, Oite T, Kitamura M. Gap junctional intercellular communication in the juxtaglomerular apparatus. Am J Physiol Renal Physiol 2009; 296: F939-F946.

[77] Yasuda K, Park HCh, Ratliff B, Addabbo F, Hatzopoulos K, Chander P, Goligorsky MS. Adriamycin nephropathy. A failure of endothelial progenitor cell-induced repair. Am J Pathol 2010; 176: 1685-1695.

[78] Yasuda K, Khandare A, Burianovskyy L, Maruyama S, Zhan F, Nasjletti A, Goligorsky MS. Tunneling nanotubes mediate rescue of prematurely senescent endothelial cells by endothelial progenitors: exchange of lysosomal pool. Aging 2011; 3: 597-608.

[79] ZANI BG, Indofli L, EdELMAN ER. Tubular bridges for bronchial epithelial cell migration and communication. PLOS ONE 2010; 5: e8930.

[80] Zhao H, Pykalainen A, Lappalainen P. I-BAR domain proteins: linking actin and plasma membrane dynamics. Curr Opin Cell Biol 2011; 23: 14-21.

Agnieszka Knopik-Srocka

Department of Cell Biology

Adam Mickiewicz University of Poznań

Umultowska 89 Street

61-614 Poznań

tel.: 0618295824

e-mail:askro@amu.edu.pl 